\title{
A third red supergiant rich cluster in the Scutum-Crux arm
}

\author{
J. S. Clark ${ }^{1}$, I. Negueruela ${ }^{2}$, B. Davies ${ }^{3,4}$, V. M. Larionov ${ }^{5,6}$, B. W. Ritchie ${ }^{1,7}$, D. F. Figer ${ }^{4}$, M. Messineo ${ }^{4}$, \\ P. A. Crowther ${ }^{8}$, and A. A. Arkharov ${ }^{9}$ \\ 1 Department of Physics and Astronomy, The Open University, Walton Hall, Milton Keynes MK7 6AA, UK \\ e-mail: S.Clark@open.ac.uk \\ 2 Departamento. de Física, Ingeniería de Sistemas y Teoría de la Señal, Universidad de Alicante, Apdo. 99, 03080 Alicante, Spain \\ 3 School of Physics \& Astronomy, University of Leeds, Woodhouse Lane, Leeds LS2 9JT, UK \\ ${ }^{4}$ Chester F. Carlson Centre for Imaging Science, Rochester Institute of Technology, 54 Lomb Memorial Drive, Rochester NY 14623 , \\ USA \\ 5 Astronomical Institute of St. Petersburg University, Petrodvorets, Universitetsky pr. 28, 198504 St. Petersburg, Russia \\ ${ }_{7}^{6}$ Isaac Newton Institute of Chile, St. Petersburg branch, Russia \\ 7 IBM United Kingdom Laboratories, Hursley Park, Winchester, Hampshire SO21 2JN, UK \\ 8 Department of Physics \& Astronomy, University of Sheffield, Sheffield S3 7RH, UK \\ 9 Pulkovo Astronomical Observatory, 196140 St. Petersburg, Russia
}

Received 25 February 2009 / Accepted 8 March 2009

\section{ABSTRACT}

\begin{abstract}
Aims. We aim to characterise the properties of a third massive, red supergiant dominated galactic cluster.
Methods. To accomplish this we utilised a combination of near/mid- IR photometry and spectroscopy to identify and classify the properties of cluster members, and statistical arguments to determine the mass of the cluster.

Results. We found a total of 16 strong candidates for cluster membership, for which formal classification of a subset yields spectral types from K3-M4 Ia and luminosities between $\log \left(L / L_{\odot}\right) \sim 4.5-4.8$ for an adopted distance of $6 \pm 1 \mathrm{kpc}$. For an age in the range of 16-20 Myr, the implied mass is $2-4 \times 10^{4} M_{\odot}$, making it one of the most massive young clusters in the Galaxy. This discovery supports the hypothesis that a significant burst of star formation occurred at the base of Scutum-Crux arm between 10-20 Myr ago, yielding a stellar complex comprising at least $\sim 10^{5} M_{\odot}$ of stars (noting that since the cluster identification criteria rely on the presence of RSGs, we suspect that the true stellar yield will be significantly higher). We highlight the apparent absence of X-ray binaries within the star formation complex and finally, given the physical association of at least two pulsars with this region, discuss the implications of this finding for stellar evolution and the production and properties of neutron stars.
\end{abstract}

Key words. stars: supergiants - Galaxy: open clusters and associations: individual: ScutumCrux arm - stars: late-type

\section{Introduction}

The vigorous star formation that characterises starburst galaxies results in the production of extended complexes of young massive stellar clusters, which span hundreds of parsecs but appear to have formed over a limited time-frame ( $\leq 20 \mathrm{Myr}$; Bastian et al. 2005). With masses $>10^{4} M_{\odot}$, analogues of such constituent clusters had been thought to be absent from our own Galaxy. However near-IR observations revealed that the Galactic Centre hosts 3 such young massive clusters (Figer et al. 1999, 2002, 2004), while detailed study of Westerlund 1 suggests a mass of the order of $10^{5} M_{\odot}$ (Clark et al. 2005). Such discoveries raise the exciting prospect of directly determining such fundamental properties as their (Initial) Mass Function; currently impossible for unresolved extragalactic examples.

Furthermore, their presence in the Galaxy permits the detailed investigation of massive stellar evolution, since their high mass yields significant numbers of rare spectral types in a co-eval setting of uniform metallicity. With ages $<5 \mathrm{Myr}$, Westerlund 1 and the Galactic Centre clusters provide valuable insights into the properties and evolutionary pathways of massive $\left(>40 M_{\odot}\right)$ stars. Recently, studies by Figer et al. (2006, F06) and Davies et al. (2007, 2008; D07 and D08 respectively) have revealed two further massive clusters dominated by red supergiants (RSGs) at the base of Scutum-Crux arm - RSGC1 $\left(12 \pm 2 \mathrm{Myr} ; M_{\text {initial }}=3 \pm 1 \times 10^{4} M_{\odot}\right)$ and RSGC2 $(17 \pm 3 \mathrm{Myr}$ $\left.M_{\text {initial }}=4 \pm 1 \times 10^{4} M_{\odot}\right)$. Collectively, both clusters sample a somewhat lower range of stellar masses, hosting 40 RSGs with $M_{\text {initial }} \sim 14-20 M_{\odot}$; of particular interest since such stars are thought to be type II SNe progenitors (Smartt et al. 2008).

In this paper we report the discovery of a third massive, RSG dominated cluster, RSGC3, also located at the base of the Scutum-Crux arm. Identified visually in GLIMPSE/Spitzer midIR images (Benjamin et al. 2003) as a concentration of bright stellar sources at $\sim l=29.2, b=-0.2$, we utilised near-IR photometry to identify potential cluster members, a subset of which were subsequently observed spectroscopically to provide a firm classification. Finally, a synthesis of these data were used to constrain the bulk properties of the cluster and individual stars within it, enabling a comparison to RSGC1 and 2 and a characterisation of the star forming environment they delineate.

\section{The RSG candidate sample}

As can be seen from the near IR images of RSGC1-3 (F06, D07, Figs. 1 and 2) it is extremely difficult to determine a physical extent for such (putative) clusters since, with the exception of the RSGs, no other cluster population is readily visible as an overdensity with respect to the stellar field population. If kinematic 


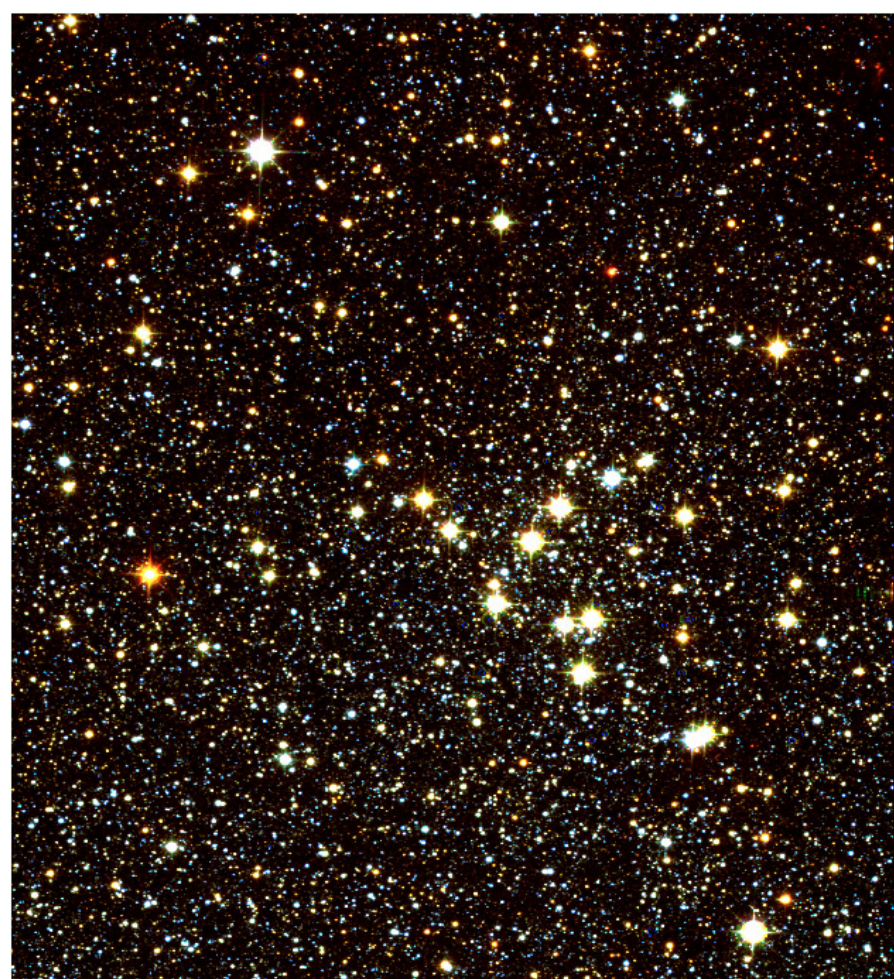

Fig. 1. Near-IR $J H K$-band colour composite of the central $\sim 7.5 \times 8.5$ of RSGC3 $(\sim 13.1 \times 14.9 \mathrm{pc}$ at a distance of $6 \mathrm{kpc})$, constructed from UKIDSS data (Lawrence et al. 2007) with artifacts due to saturation artificially removed. Note the lack of a clearly defined stellar overdensity of unevolved cluster members with respect to the field.

information is available, it is possible to identify a co-moving, physical association of RSGs, in order to discriminate between cluster and field stars (e.g. D07). However, the spectroscopic data presented here are of insufficient resolution to extract the radial velocity of cluster members, while, unlike RSGC1 and 2 (F06; D07), we find no maser emission from any cluster members which would also provide kinematic constraints (Verheyen et al. in prep.); thus we are forced to utilise photometric data to construct a list of candidate cluster members.

Based on the spatial concentration of bright red stars, we start by taking 2MASS photometry for stars within $r \leq 3^{\prime}$ of the position of Star 1 (RA: $18^{\mathrm{h}} 45^{\mathrm{m}} 23.60^{\mathrm{s}}$, Dec: $-03^{\circ} 24^{\prime} 13^{\prime \prime}$ '9), selecting only stars with quality flags "AAA" and error $\Delta K_{\mathrm{S}} \leq$ 0.05 . The ten bright stars defining the spatial concentration form a well-separated group in the $\left(J-K_{\mathrm{S}}\right) / K_{\mathrm{S}}$ diagram (Fig. 3), around $\left(J-K_{\mathrm{S}}\right) \approx 3.0$. This grouping is also present in the $(H-$ $\left.K_{\mathrm{S}}\right) / K_{\mathrm{S}}$ diagram, centred around $\left(H-K_{\mathrm{S}}\right)=0.95$. We then calculate the reddening-free parameter $Q_{\mathrm{IR}}=(J-H)-1.8 \times\left(H-K_{\mathrm{S}}\right)$. Early-type stars have $Q_{\mathrm{IR}} \approx 0.0$, while most bright field stars have $Q_{\mathrm{IR}} \approx 0.4-0.5$, corresponding to red giants (Indebetouw et al. 2005; Negueruela \& Schurch 2007). All ten stars form a clearly separated grouping in this diagram, with values $0.2-0.4$, typical of supergiants. There is one more star in this clump, S14, which has redder $\left(J-K_{\mathrm{S}}\right)$ and $\left(H-K_{\mathrm{S}}\right)$. The only other star of comparable $K_{\mathrm{S}}$ in the field, $\mathrm{S} 28$, has $Q_{\mathrm{IR}}=0.08$, typical of an early-type star. Considering the large number of bright stars in the field and the spatial extent of other starburst clusters in the area (e.g. F06, D07), we extended the search to $r \leq 7^{\prime}$. The group in the $\left(J-K_{\mathrm{S}}\right) / K_{\mathrm{S}}$ and $\left(H-K_{\mathrm{S}}\right) / K_{\mathrm{S}}$ diagrams, which we consider to comprise prime cluster members now includes $\mathrm{S} 11$, S12, S13 and S15 (Table 1).

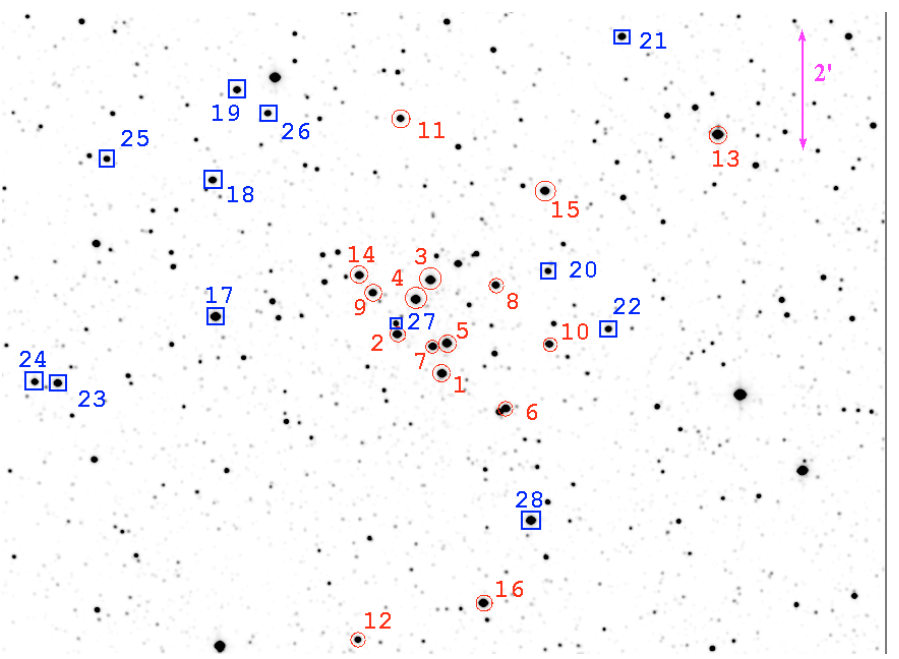

Fig. 2. Finding chart for RSGC3, with the stars listed in Table 1 indicated. The finder comprises a $K_{\mathrm{s}}$-band image from 2MASS with a $14^{\prime} \times 10^{\prime}$ field of view centred on the cluster $(\sim 24.5 \times 17.5 \mathrm{pc}$ for a distance of $6 \mathrm{kpc})$. Note that this represents a larger field of view than the near-IR image presented in Fig. 1. The circles represent stars we consider to be bona fide cluster members, while the squares indicate the remaining stars discussed in the text, a subset of which (S17-22) are also likely cluster members (see Sect. 3 for details).

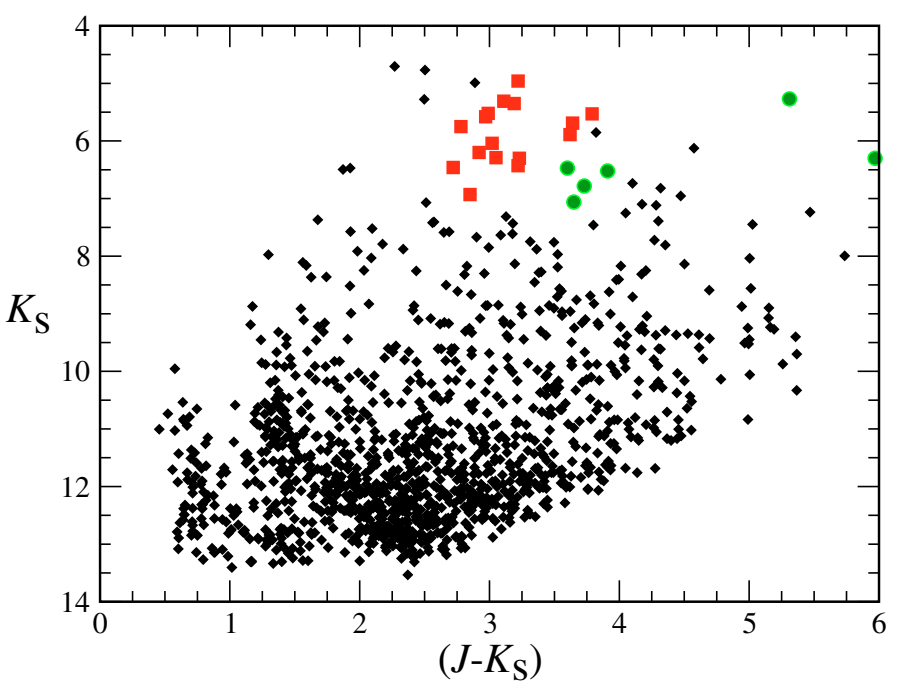

Fig. 3. Colour magnitude plot for stars within 7 ' of RSGC3. The 16 cluster members identified in Sect. 3 are indicated by the red squares, the remaining 6 likely cluster members (Sect. 2 and Table 1) are plotted as green circles. Note that the two outlying stars with $\left(J-K_{\mathrm{S}}\right)>5.0$ are S17 and S21, discussed in Sect. 3. Finally, of the two stars which are also located with the (likely) cluster members and not marked as such, one is a blend and hence we exclude it from further analysis, while the second is $\mathrm{S} 23$, which has a discrepant $\left(H-K_{\mathrm{S}}\right)$ index and $Q_{\mathrm{IR}}=-0.4$, suggesting that it may be an emission line star rather than a RSG.

A number of objects - S14 (found in the cluster core), S15-16 and S18-22 - have $Q_{\mathrm{IR}}$ similar to the above, but with redder $-\left(J-K_{\mathrm{S}}\right) \approx 3.7$ - colours; the separation between these stars and the main plume of red giant stars in terms of $Q_{\mathrm{IR}}$ is not as well defined as that for the prime cluster candidates. We identify these as likely cluster members, with the difference in colours potentially due to excess reddening with respect to the core members (noting that significant differential reddening is also observed for RSGC2; D07). Finally, for completeness, with the inclusion of S23 and S27, these stars form a well defined 
Table 1. Summary of RSG candidates and their properties. Top panel: the core group of 15 photometrically selected stars regarded as prime cluster candidates; second panel: likely cluster members; third panel: stars of interest, as defined in Sect. 2 and fourth panel: likely foreground RSG identified by spectroscopy and included for completeness. Note that based on spectroscopy, we also consider it likely that star S16 is a cluster member and treat it as such in the text. Co-ordinates and near-IR magnitudes are from 2MASS, with mid-IR $(\sim 5-25 \mu \mathrm{m})$ magnitudes from the Galactic plane surveys of GLIMPSE/Spitzer (Benjamin et al. 2003) and the Midcourse Source Experiment (MSX) (Egan et al. 2001). We also provide the dereddened $M S X(A-C)$ colour (adopting the prescription of Messineo et al. 2005), which is a diagnostic of emission from circumstellar dust (and hence proxy for mass loss) and the spectral type of the stars, where available (Sect. 3).

\begin{tabular}{|c|c|c|c|c|c|c|c|c|c|c|c|c|c|}
\hline \multirow[t]{2}{*}{ ID } & \multicolumn{2}{|c|}{ " Co-ordinates (J2000) } & \multicolumn{3}{|c|}{ 2MASS } & \multirow{2}{*}{$\begin{array}{c}\text { GLIMPSE } \\
5.8 \mu \mathrm{m}\end{array}$} & \multirow{2}{*}{$\begin{array}{l}\text { Spitzer } \\
8.0 \mu \mathrm{m}\end{array}$} & \multirow[b]{2}{*}{ A } & \multicolumn{3}{|c|}{$M S X$} & \multirow[b]{2}{*}{$(A-C)$} & \multirow{2}{*}{$\begin{array}{l}\text { Spec. } \\
\text { type }\end{array}$} \\
\hline & RA & Decln. & $J$ & $H$ & $K_{\mathrm{s}}$ & & & & $\mathrm{C}$ & $\mathrm{D}$ & $\mathrm{E}$ & & \\
\hline S1 & 184523.60 & -032413.9 & 8.55 & 6.54 & 5.58 & 4.73 & 4.50 & - & - & - & - & - & RSG \\
\hline S2 & 184526.54 & -032335.3 & 8.53 & 6.62 & 5.75 & 4.92 & 4.63 & 4.30 & 3.39 & - & - & - & M3 Ia \\
\hline S3 & 184524.34 & -032242.1 & 8.54 & 6.43 & 5.35 & 4.19 & 4.14 & - & - & - & - & - & M4 Ia \\
\hline $\mathrm{S} 4$ & 184525.31 & -032301.1 & 8.42 & 6.39 & 5.31 & 4.08 & 4.04 & 3.97 & 3.12 & 3.21 & - & 0.85 & M3 Ia \\
\hline S5 & 184523.26 & -032344.1 & 8.51 & 6.52 & 5.52 & 4.45 & 4.20 & 3.78 & 2.82 & 3.15 & - & 0.96 & M2 Ia \\
\hline S6 & 184519.39 & -032448.3 & 9.06 & 6.97 & 6.04 & 5.24 & 5.20 & 4.71 & - & - & - & - & RSG \\
\hline S7 & 184524.18 & -032347.3 & 9.12 & 7.10 & 6.20 & 5.22 & 4.84 & - & - & - & - & - & M0 Ia \\
\hline S8 & 184520.06 & -032247.1 & 9.53 & 7.29 & 6.30 & 5.46 & 5.43 & 5.60 & - & - & - & - & K5 Ia \\
\hline S9 & 184528.13 & -032254.6 & 9.34 & 7.26 & 6.29 & 5.49 & 5.44 & - & - & - & - & - & M0 Ia \\
\hline $\mathrm{S} 10$ & 184516.56 & -032345.1 & 9.65 & 7.43 & 6.43 & 5.57 & 5.52 & 5.45 & - & - & - & - & M0 Ia \\
\hline S11 & 184526.31 & -03 2003.3 & 9.18 & 7.27 & 6.46 & 5.77 & 5.78 & 5.87 & - & - & - & - & RSG \\
\hline $\mathrm{S} 12$ & 184529.12 & -032835.6 & 9.78 & 7.86 & 6.93 & 6.57 & 6.26 & 6.23 & - & - & - & - & - \\
\hline $\mathrm{S} 13$ & 184505.51 & -03 2019.6 & 8.18 & 5.97 & 4.96 & - & - & 3.59 & 2.43 & 2.45 & - & 1.16 & RSG \\
\hline S14 & 184529.02 & -03 2237.4 & 9.51 & 7.08 & 5.89 & 4.79 & 4.61 & 4.43 & - & - & - & - & RSG \\
\hline $\mathrm{S} 15$ & 184516.84 & -032114.6 & 9.33 & 6.92 & 5.69 & 4.12 & - & 3.26 & 1.85 & 1.66 & 0.92 & 1.41 & RSG \\
\hline S16 & 184520.87 & -032759.6 & 9.32 & 6.78 & 5.53 & 4.34 & 4.15 & 3.79 & 2.93 & 2.97 & 2.22 & 0.86 & RSG \\
\hline S17 & 184538.45 & -032318.1 & 10.58 & 7.07 & 5.27 & - & - & 2.09 & 0.65 & 0.45 & -0.121 & 1.44 & - \\
\hline S18 & 184538.65 & -03 2103.9 & 10.07 & 7.62 & 6.47 & 5.50 & 5.44 & 5.36 & - & - & - & - & - \\
\hline S19 & 184537.05 & $-03 \quad 1935.1$ & 10.43 & 7.81 & 6.52 & 5.44 & 5.40 & 5.42 & - & - & - & - & - \\
\hline S20 & 184516.64 & -032233.2 & 10.71 & 8.28 & 7.06 & 5.98 & 5.85 & 5.79 & - & - & - & - & - \\
\hline S21 & 184511.81 & -031843.1 & 12.27 & 8.30 & 6.30 & 4.50 & 4.32 & 4.27 & 3.31 & 3.17 & - & 0.96 & - \\
\hline S22 & 184512.69 & -032330.7 & 10.51 & 8.04 & 6.78 & 5.49 & 5.41 & 5.36 & - & - & - & - & - \\
\hline S23 & 184548.82 & -032423.1 & 9.67 & 7.36 & 5.85 & - & - & 2.92 & 2.00 & 1.84 & 1.27 & 0.92 & - \\
\hline $\mathrm{S} 24$ & 184550.34 & -032421.8 & 10.84 & 8.08 & 6.74 & - & - & - & - & - & - & - & - \\
\hline S25 & 184545.61 & -032042.8 & 11.27 & 8.43 & 7.10 & 5.88 & 5.96 & - & - & - & - & - & - \\
\hline S26 & 184535.02 & -031958.2 & 11.43 & 8.43 & 6.96 & 5.42 & 5.21 & - & - & - & - & - & - \\
\hline S27 & 184526.61 & -032324.7 & 11.26 & 8.78 & 7.46 & 6.01 & 5.81 & - & - & - & - & - & - \\
\hline S28 & 184517.75 & -032638.4 & 7.78 & 6.14 & 5.38 & 4.42 & 4.17 & 3.18 & 3.12 & - & - & 1.19 & RSG \\
\hline
\end{tabular}

group in the $\left(J-K_{\mathrm{S}}\right) / K_{\mathrm{S}}$ diagram. However, only S14, S15, S16 and $\mathrm{S} 18$ are grouped in the $\left(H-K_{\mathrm{S}}\right) / K_{\mathrm{S}}$, while $\mathrm{S} 19, \mathrm{~S} 20, \mathrm{~S} 22$, $\mathrm{S} 24, \mathrm{~S} 25, \mathrm{~S} 26$ and S27 form a second, distinct group in $\left(H-K_{\mathrm{S}}\right)$. Therefore, given their magnitudes and red colours, we identify S23-27 as potential objects of interest, but as with our second group, they require spectroscopic follow up to ascertain their nature and relationship to RSGC3 (Table 1).

To summarise, based solely on their near-IR properties we identify a core group of 15 prime candidate cluster members, a second group of 7 likely cluster members, and a final group of 5 bright red stars that deserve investigation within $r \leq 7^{\prime}$ of the nominal cluster core (Table 1).

\section{Spectroscopic results and analysis}

Based on their photometric properties, initial low resolution $(R \sim 270)$ observations of 17 stars were made with the IR imaging camera SWIRCAM+HK grism, mounted on the AZT-24 $1.1 \mathrm{~m}$ telescope at Campo Imperatore on 2006 September 3 and 4. Subsequently, higher resolution observations of 8 targets, made in the flexible observing mode, were obtained with the $1-5 \mu$ m imaging spectrometer UIST, mounted on the United Kingdom Infra-Red Telescope on 2007 June 8 and 21 (Program
ID U/07A/15). The Long K grism was used with the 4-pixel slit, giving wavelength coverage from $2.20-2.51 \mu \mathrm{m}$ with a resolution $R \sim 1900$. Data reduction was accomplished via the methodology described in Clark et al. (2003), and the spectra are presented in Fig. 2.

Of the 17 low resolution spectra, 16 show deep CO bandhead absorption, characteristic of late type stars (Fig. 4). Of these, 14 are photometrically defined core cluster members; one, S16, a likely member, and the final star, S28, appears to be a foreground object based on its near-IR colours (Table 1). Following the methodology of F06 and D07, it is possible to use the strength of the $\mathrm{CO}$ bandheads to provide a spectral and luminosity classification for the stars. However this requires a robust determination of the stellar continuum, which proved impossible for the low resolution spectra, and consequently was only attempted for the subset of 8 stars for which medium resolution data were available.

We find all 8 stars - S2-5 and S7-10 - to be supergiants, with spectral types ranging from K5-M4 Ia; the resultant temperatures (and associated errors) are summarised in Table 2. Given the equivalence of the low resolution spectra of these stars with those of S1, S6, S1 1 and S13-16, we conclude that these stars are likewise RSGs. Thus these results provide strong support for the 


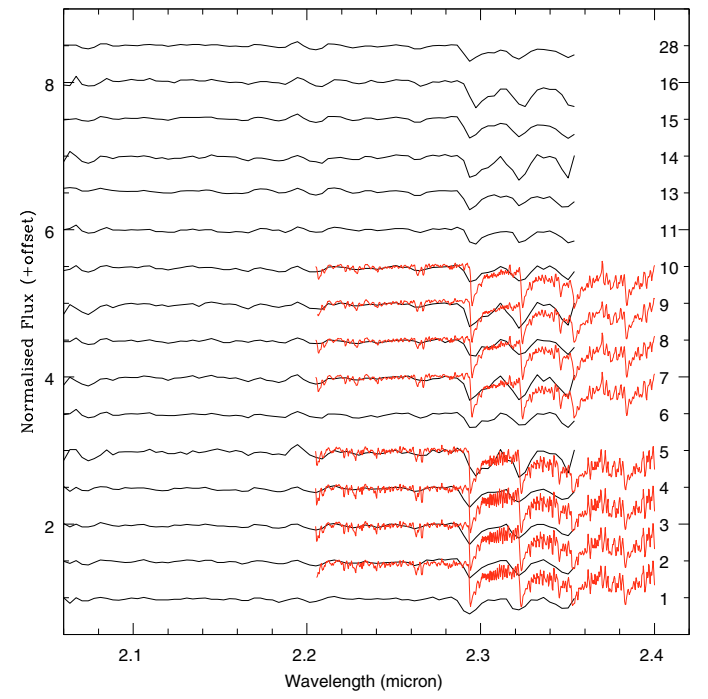

Fig. 4. Montage of low (Campo Imperatore; black) and medium resolution (UKIRT; red) spectra of selected photometric targets, revealing the prominent $\mathrm{CO}$ bandhead absorption.

Table 2. Summary of the stellar properties of the 8 RSGs for which accurate spectral classification was possible, assuming a cluster distance of $6 \mathrm{kpc}$.

\begin{tabular}{lccccc}
\hline \hline ID\# & $\begin{array}{c}T_{\text {eff }} \\
(\mathrm{K})\end{array}$ & $\begin{array}{c}\text { Spec } \\
\text { type }\end{array}$ & $A_{K}$ & $M_{K}$ & $\log \left(L_{\mathrm{bol}} / L_{\odot}\right)$ \\
\hline 2 & $3605 \pm 147$ & M3 & $1.20 \pm 0.12$ & $-9.34_{-0.34}^{+0.39}$ & $4.51_{-0.15}^{+0.14}$ \\
3 & $3535 \pm 125$ & M4 & $1.47 \pm 0.08$ & $-10.09_{-0.33}^{+0.37}$ & $4.79_{-0.15}^{+0.13}$ \\
4 & $3605 \pm 147$ & M3 & $1.44 \pm 0.12$ & $-10.12_{-0.34}^{+0.39}$ & $4.82_{-0.15}^{+0.14}$ \\
5 & $3660 \pm 130$ & M2 & $1.43 \pm 0.07$ & $-9.85_{-0.32}^{+0.37}$ & $4.73_{-0.15}^{+0.13}$ \\
7 & $3790 \pm 130$ & M0 & $1.40 \pm 0.11$ & $-9.11_{-0.33}^{+0.38}$ & $4.48_{-0.15}^{+0.13}$ \\
8 & $3840 \pm 175$ & K5 & $1.60 \pm 0.04$ & $-9.23_{-0.31}^{+0.36}$ & $4.54_{-0.14}^{+0.12}$ \\
9 & $3790 \pm 130$ & M0 & $1.53 \pm 0.11$ & $-9.14_{-0.33}^{+0.38}$ & $4.49_{-0.15}^{+0.13}$ \\
10 & $3790 \pm 130$ & M0 & $1.60 \pm 0.11$ & $-9.09_{-0.33}^{+0.38}$ & $4.47_{-0.15}^{+0.13}$ \\
\hline
\end{tabular}

identification of S1-15 as bona fide cluster members based on both spectroscopic and photometric criteria, with S16 possibly a more heavily reddened cluster member. For the remainder of the paper we therefore count these sixteen stars as cluster members; in a future paper we will use high resolution spectroscopy to confirm such a physical association (Davies et al. in prep.).

The current lack of kinematic data precludes the determination of the cluster distance via comparison to the Galactic rotation curve, and hence the luminosity, age and initial mass of the cluster members (since RSGs span a wide range of luminosities $\left(\log \left(L_{\mathrm{bol}} / L_{\odot}\right) \sim 4.0-5.8\right.$; Meynet \& Maeder 2000). Nevertheless, we find a mean value of $A_{K_{S}} \sim 1.5$, from which we may infer $A_{V} \sim 13.0$ and hence, assuming a canonical $1.8 \mathrm{mag}$. extinction per kpc, an upper limit to the distance to RSGC3 of $\sim 7.2 \mathrm{kpc}$ (Rieke \& Lebofsky 1985; Egan et al. 2002). Such a value is entirely consistent with a location of RSGC3 at a similar distance to RSGC1 and 2 at the end of the Galactic Bar $(6.60 \pm 0.89 \mathrm{kpc}$ and $5.83_{-0.76}^{1.91} \mathrm{kpc}$ respectively, D08); we thus adopt a distance of $6 \pm 1 \mathrm{kpc}$ for the remainder of this work. At such a distance, utilising the temperature/spectral type calibration and resultant bolometric corrections of Levesque et al. (2005), we find $\log \left(L_{\mathrm{bol}} / L_{\odot}\right) \sim 4.5-4.8$ for S2-5 and 7-10 and corresponding ages and initial masses of 16-20 Myr and $\sim 10-13 M_{\odot}$ (Fig. 5).

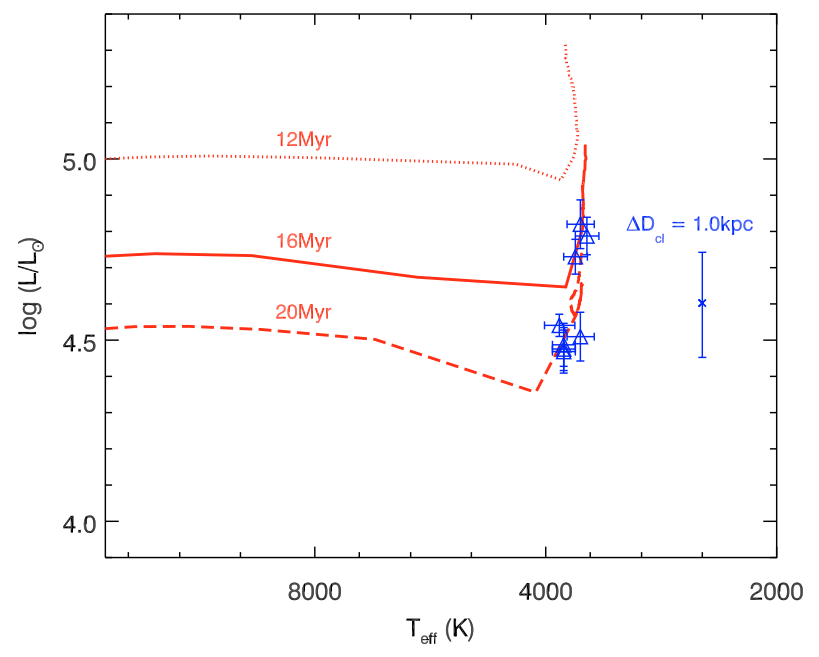

Fig. 5. H-R diagram showing the locations of the 8 RSGs for which accurate spectral classification was possible assuming a distance to the cluster of $D_{\mathrm{cl}} \sim 6 \mathrm{kpc} .12,16$ and $20 \mathrm{Myr}$ isochrones from the rotating models of Meynet \& Maeder (2000) have been overplotted. Errors on the data points do not include the uncertainty in the cluster distance $\left(\Delta D_{\mathrm{cl}}\right)$; representative error-bars assuming an uncertainty of $\pm 1 \mathrm{kpc}$ are indicated to the right of the figure.

Finally, the observed range of the dereddened [8]-[12] colour index $-M S X(A-C) \sim 0.85-1.44$ (Table 1) - for the 7 (candidate) cluster members for which it may be determined is directly comparable to that found by D07 for RSGC2. Sampling the broad silicate emission feature, this provides a measure of the mass loss rate via the dust content of the circumstellar environment. It is therefore of interest that both of the stars with discrepant $\left(J-K_{\mathrm{S}}\right)$ colours show excesses (S17 and S12; Fig. 3), suggesting that a build-up of circumstellar material due to enhanced mass-loss affects their near-IR properties, such as is observed in RSGC2-49 (D07).

Following the methodology pioneered by F06, we utilise Monte Carlo simulations, employing rotating stellar models (D07) to estimate the initial mass of the cluster from the number of RSGs currently present. For ages of 16(20) Myr this yields masses of $2(4) \times 10^{4} M_{\odot}$. If our second tier of RSG candidates are confirmed as cluster members, the mass estimate would increase by $\sim 30 \%$, noting that this would not be altered by increasing the distance to a maximum of $7 \mathrm{kpc}$ (as implied by the reddening). Finally, since stellar evolutionary codes predict a spread in intrinsic RSG luminosities for even co-eval clusters, the observed range of $\log \left(L_{\mathrm{bol}} / L_{\odot}\right) \sim 4.5-4.8$ for cluster members should not be interpreted as implying non-coevality.

\section{Discussion and concluding remarks}

With an age of 16-20 Myr and a total mass of 2-4 $\times 10^{4} \mathrm{M}_{\odot}$, RSCG3 appears to be a close counterpart to RSGC1 and 2 (D08), while the properties of the constituent stars in terms of spectral types, luminosities and circumstellar environments are also directly comparable to the members of those clusters. As such RSGC3 belongs to an increasing population of hitherto unsuspected young massive clusters within the Galaxy. With ages ranging from 2-3 Myr for the Arches to 16-20 Myr for RSGC2 and 3 they also provide a fertile testbed for constraining the lifecycle of stars of $\sim 14 M_{\odot}$ and above.

Additionally, the proximity of RSGC3 to both RSGC1 and 2 - projected distances of $\sim 400 \mathrm{pc}$ and $\sim 300 \mathrm{pc}$ respectively for 
$d=6 \mathrm{kpc}-$ at the base of Scutum-Crux arm $\left(l \sim 25-29^{\circ}\right)$ provides strong support for the hypothesis that this region has been subject to a recent burst of star formation (Garzón et al. 1997, D07), yielding an extended stellar cluster complex such as those observed in external galaxies such as M51 (Bastian et al. 2005). If correct, the "starburst" has yielded a total of $>9 \times 10^{4} M_{\odot}$ of stars just considering the 3 clusters. However, D07 report the presence of an additional population of RSGs in the vicinity of RSGC2, while Garzón et al. (1997) and López-Corredoira et al. (1999) also report a significant "diffuse" field population of cool supergiants within the region delineated by RSGC1-3, suggesting that the true total may be significantly higher.

Consideration of the near-IR images of RSGC1-3 emphasises this possibility; the clusters are only identifiable as such due to their significant RSG populations, as no other overdensity of (less evolved) stars is apparent. However, such stars are intrinsically short lived and consequently rare, and so only signpost the location of clusters for which the (unevolved) stellar population is unresolvable against the field for a narrow range of cluster masses and ages. For instance, for clusters with masses of the order of $10^{3} M_{\odot}$ (so comparable to the Orion cluster) one would only expect the presence of $\sim 1-3$ RSGs at any given epoch on statistical grounds, suggesting that such clusters would be difficult to identify in a near-IR survey (a problem even afflicting more massive clusters for ages $<10 \mathrm{Myr}$; D07).

Likewise, for clusters with ages of over $20 \mathrm{Myr}$, while one would expect a large number of red evolved stars to be present, their lower intrinsic luminosity will make them more difficult to discriminate against the field in any search for cluster candidates. Furthermore, the rapid dissolution of clusters due to ejection of the intercluster medium by stellar winds and $\mathrm{SNe}$ (Goodwin \& Bastian 2006) exacerbates this problem, reducing the spatial density of any RSGs within clusters. An additional result of this process is that the velocity dispersion of cluster members will increase, in turn magnifying the uncertainty in the identification of a physical association via kinematic means.

Therefore, while the presence of RSGC1-3 points to an episode of enhanced star formation 10-20 Myr ago, from the methodology employed in this work it is difficult to determine whether there was significant activity before this date or indeed what the total stellar yield of this "starburst" was. An analogous argument applies to determining the star formation history for ages $<10 \mathrm{Myr}$, although the lack of Giant H II regions within this putative complex (Conti \& Crowther 2004) implies that no massive clusters are currently forming.

We caution that these limitations will manifest themselves in any other near-IR searches for stellar clusters in regions of the disc with a high (projected) stellar density, likely leading to significant incompleteness in any Galactic cluster mass function determined via such a methodology.

\subsection{Association with X-ray binaries and post SNe compact objects}

Given a potential SNe rate of one every 40-80 kyr (F06), and the likely association of two pulsars with RSGC1 (F06, Gotthelf \& Halpern 2008) we examined the catalogues of Liu et al. (2006) and Bird et al. (2007) to search for any relativistic sources associated with RSGC3, but found none. Motivated by the hypothesis that RSGC1-3 delineate a star forming complex, we extended this search to this region $\left(l=25-30^{\circ}, b= \pm 1^{\circ}\right)$, but again found no accreting X-ray binaries within it for a distance of $6 \pm 1 \mathrm{kpc}$. Given the increasing evidence for a high binary fraction amongst massive stars (e.g., Clark et al. 2008), the absence of any Be/X-ray binaries - systems consisting of a B0-3 V-IIIe primary and a neutron star accretor - is surprising, since one would expect them to be active at such an epoch (10-20 Myr; e.g., Portegies Zwart \& Verbunt 1996). Nevertheless, one might suppose that a combination of their transient nature plus a $\mathrm{SNe}$ kick sufficient to either disrupt or rapidly eject a surviving binary from the complex may explain their lack of detection.

However, we note with interest the location of the Anomalous X-ray Pulsar (AXP) AX J1841.3-0455; $l=27.39$, $b=-0$ d006. Durant \& van Kerkwijk (2006) estimate a lower limit of $>5 \mathrm{kpc}$ to AX J1841.3-0455, while Vasisht \& Gotthelf (1997) provide an upper limit of $7 \mathrm{kpc}$ from its association with the SNR Kes 73. Taken together they raise the possibility that it could be physically associated with the putative star formation complex, with AX J1841.3-0455 located equidistantly between RSGC2 and 3, and directly within the region identified by Garzón et al. (1997) and López-Corredoira et al. (1999) as showing a significant overdensity of "field" RSGs. The expected rate of SNe for such a complex would be fully consistent with the relative youth expected of magnetars (Thompson et al. 2000), while SGR 1900+14 demonstrates that their progenitors can have masses as low as $\sim 15 M_{\odot}$ (Clark et al. 2008; Davies et al. in prep.), also consistent with the current RSG population at the base of the Scutum Crux arm.

Definitively associating AX J1841.3-0455 with the same burst of star formation that yielded RSG1-3 would imply a progenitor mass of $<20 M_{\odot}$, and hence provide additional evidence that the hypothesis that high-mass stars are required to produce magnetars is incorrect. Moreover, consideration of SGR 1900+14, AX J1838.0-0655 (the young pulsar associated with RSGC1) and potentially AX J1841.3-0455 suggests that despite having progenitors of comparable mass $\left(\sim 15-18 M_{\odot}\right.$; Clark et al. 2008, D08, Davies et al. in prep.), the surface magnetic fields of the resultant neutron stars can differ by over two orders of magnitude (Gotthelf \& Halpern 2008; Kouveliotou et al. 1999; Vasisht \& Gotthelf 1997), presumably reflecting differences in the properties of their progenitors other than, or being directly dependant on, stellar mass (such as magnetic field or rotational velocity).

Acknowledgements. J.S.C. acknowledges support from an RCUK fellowship, and thanks Sophie Allen for assistance in the preparation of Fig. 1, and Mike \& Tessa Allen for their kind hospitality during the production of this paper. This research is partially supported by the Spanish Ministerio de Ciencia e Innovación (MICINN) under grants AYA2008-06166-C03-03 and CSD2006-70. AZT-24 observations are made within an agreement between Pulkovo, Rome and Teramo observatories D.F. acknowledges support from NASA under award NNG 05-GC37G, through the Long-Term Space Astrophysics program and from NYSTAR under a Faculty Development Program grant. The UKIDSS project is defined in Lawrence et al. (2007). UKIDSS uses the UKIRT Wide Field Camera (Casali et al. 2007). The photometric system and calibration are described in Hewett et al. (2006) and Hodgkin et al. (2008), with pipeline processing and archiving described in Irwin et al. (in prep.) and Hambly et al. (2008). This paper makes use of data products from the Two Micron All Sky Survey, which is a joint project of the University of Massachusetts and the Infrared Processing and Analysis Center/California Institute of Technology, funded by the National Aeronautics and Space Administration and the National Science Foundation.

Note added in proof. Shortly after acceptance we became aware of the co-discovery of this cluster by Alexander et al. (2009).

\section{References}

Alexander, M. J., Kobulnicky, H. A., Clemens, D. P., et al. 2009, AJ, in press [arXiv:0903.2496]

Bastian, N., Gieles, M., Efremov, Yu. N., \& Lamers, H. J. G. L. M. 2005, A\&A, 443, 79

Benjamin, R. A., Churchwell, E., Babler, B. L., et al. 2003, PASP, 115, 953 
Bird, A. J., Malizia, A., Bazzao, A., et al. 2007, ApJS, 170, 175

Casali, M., Adamson, A., \& Alves de Oliviera, C. 2007, A\&A, 467, 777

Clark, J. S., Larionov, V. M., Crowther, P. A., Egan, M. P., \& Arkharov, A. 2003, A\&A, 403, 653

Clark, J. S., Negueruela, I., Crowther, P. A., \& Goodwin, S. P. 2005, A\&A, 434, 949

Clark, J. S., Muno, M. P., Negueruela, I., et al. 2008, A\&A, 477, 147

Conti, P. S., \& Crowther, P. A. 2004, MNRAS, 355, 899

Davies, B., Figer, D. F., Kudritzki, R.-F., et al. 2007, ApJ, 671, 781 (D07)

Davies, B., Figer, D. F., Law, C. J., et al. 2008, ApJ, 676, 1016 (D08)

Durant, M., \& van Kerkwijk, M. H. 2006, ApJ, 650, 1070

Egan, M. P., Price, S. D., \& Gugliotti, G. M. 2001, BAAS, 34, 561

Egan, M. P., Clark, J. S., \& Mizuno, D. R. 2002, ApJ, 572, 288

Figer, D. F., McLean, I. S., \& Morris, M. 1999, ApJ, 514, 202

Figer, D. F., Najarro, F., Gilmore, D., et al. 2002, ApJ, 581, 258

Figer, D. F., Rich, R. M., Kim, S. S., Morris, M., \& Serabyn, E. 2004, ApJ, 601, 319

Figer, D. F., MacKenty, J. W., Robberto, M., et al. 2006, ApJ, 643, 1166 (F06) Garzón, F., Lopez-Corredoira, M., Hammersley, P., et al. 1997, ApJ, 491, L31

Goodwin, S. P., \& Bastian, N. 2006, MNRAS, 369, L9

Gotthelf, E. V., \& Halpern, J. P. 2008, ApJ, 681, 515
Hambly, N. C., Collins, R. S., \& Cross, N. J. G. 2008, MNRAS, 384, 637 Hewett, P. C., Warren, S. J., Leggett, S. K., \& Hodgkin, S. T. 2006, MNRAS, 367,454

Hodgkin, S. T., Irwin, M. J., Hewett, P. C., \& Warren, S. J. 2008 [arXiv0812.3081]

Indebetouw, R., Mathis, J. S., \& Babler, B. L. 2005, ApJ, 619, 931

Kouveliotou, C., Strohmayer, T., Hurley, K., et al. 1999, ApJ, 510, L115

Lawrence, A., Warren, S. J., Almaini, O., et al. 2007, MNRAS, 379, 1599

Levesque, E. M., Massey, P., Olsen, K. A. G., et al. 2005, ApJ, 628, 973

Liu, Q. Z., van Paradijs, J., \& van den Heuvel, E. P. J. 2006, A\&A, 455, 1165 López-Corredoira, M., Garzón, F., Beckman, J. E., et al. 1999, AJ, 118, 381

Messineo, M., Habing, H. J., Menten, K. M., et al. 2005, A\&A, 435, 575

Meynet, G., \& Maeder, A. 2000, A\&A, 361, 101

Negueruela, I., \& Schurch, M. P. E. 2007, A\&A, 461, 631

Portegies Zwart, S. E., \& Verbunt, F. 1996, A\&A, 309, 179

Rieke, G. H., \& Lebofsky, M. J. 1985, ApJ, 288, 618

Smartt, S. J., Eldridge, J. J., Crockett, R. M., \& Maund, J. R. 2008 [arXiv0809.0403]

Thompson, C., Duncan, R. C., Woods, P. M., et al. 2000, ApJ, 543, 340

Vasisht, G., \& Gotthelf, E. 1997, ApJ, 486, L129 